This item was submitted to Loughborough's Research Repository by the author.

Items in Figshare are protected by copyright, with all rights reserved, unless otherwise indicated.

\title{
Quantum metamaterial without local control
}

PLEASE CITE THE PUBLISHED VERSION

http://dx.doi.org/10.1103/PhysRevB.87.235410

\section{PUBLISHER}

(C) American Physical Society

\section{VERSION}

VoR (Version of Record)

\section{PUBLISHER STATEMENT}

This work is made available according to the conditions of the Creative Commons Attribution-NonCommercialNoDerivatives 4.0 International (CC BY-NC-ND 4.0) licence. Full details of this licence are available at: https://creativecommons.org/licenses/by-nc-nd/4.0/

\section{LICENCE}

CC BY-NC-ND 4.0

\section{REPOSITORY RECORD}

Shvetsov, A., A.M. Satanin, Franco Nori, Sergey Savel'ev, and Alexandre M. Zagoskin. 2019. "Quantum Metamaterial Without Local Control”. figshare. https://hdl.handle.net/2134/18527. 


\title{
Quantum metamaterial without local control
}

\author{
A. Shvetsov, ${ }^{1,}{ }^{*}$ A. M. Satanin, ${ }^{1}$ Franco Nori, ${ }^{2,3}$ S. Savel'ev, ${ }^{3,4}$ and A. M. Zagoskin ${ }^{3,4, \dagger}$ \\ ${ }^{1}$ Nizhny Novgorod State University, Gagarin Avenue, 23, 603950, Nizhny Novgorod, Russia \\ ${ }^{2}$ Department of Physics, University of Michigan, Ann Arbor, Michigan 48109-1040, USA \\ ${ }^{3}$ CEMS, RIKEN, Saitama 351-0198, Japan \\ ${ }^{4}$ Department of Physics, Loughborough University, Loughborough LE11 3TU, United Kingdom
}

(Received 14 March 2013; published 10 June 2013)

\begin{abstract}
A quantum metamaterial can be implemented as a quantum coherent one-dimensional array of qubits placed in a transmission line. The properties of quantum metamaterials are determined by the local quantum state of the system. Here we show that a spatially periodic quantum state of such a system can be realized without direct control of the constituent qubits, by their interaction with the initializing ("priming") pulses sent through the system in opposite directions. The properties of the resulting quantum photonic crystal are determined by the choice of the priming pulses. This proposal can be readily generalized to other implementations of quantum metamaterials.
\end{abstract}

DOI: 10.1103/PhysRevB.87.235410

PACS number(s): $81.05 . \mathrm{Xj}, 74.81 . \mathrm{Fa}$

\section{INTRODUCTION}

Since the pioneering papers, ${ }^{1-3}$ the investigation of metamaterials (quasicontinuous media built of artificial unit elements, that modify the properties of propagating electromagnetic waves) was driven by their unusual optical properties ${ }^{4,5}$ in a wide range of frequencies. They are used to produce antireflection coating and optical devices such as adaptive lenses, reconfigurable mirrors, converters, etc. Another direction of research is related to the development of metamaterials based on active units, e.g., Josephson junctions. ${ }^{6,7}$ The emission of terahertz radiation due to the ac Josephson effect allows to build Josephson-based emitters, filters, detectors, and waveguides operating in this spectral range that is important for applications. ${ }^{8,9}$ The nonlinear effective inductance of Josephson junctions makes them a convenient basis for nonlinear metamaterials. ${ }^{10}$

Another fundamentally novel class of artificial media are quantum metamaterials, i.e., optical media, which maintain global quantum coherence over times exceeding the signal transition time and allow local control over quantum states and basic properties of their constituent elements. ${ }^{11}$ The term reflects the fact that, similarly to classical metamaterials, these systems allow additional ways to control the propagation of electromagnetic fields not available to standard materials. The optical properties of the material are determined by its controllable coherent quantum dynamics.

Recently, various schemes of qubit-array control have been proposed. ${ }^{12-14}$ Quantum metamaterials were introduced in Ref. 11 as an array of superconducting charge qubits placed inside a transmission line; the extensions to other implementations, including those in optical range, soon followed. ${ }^{15-23}$ In particular, experiments on a flux qubit inside a transmission line reproduced a number of atomic spectroscopy effects. ${ }^{24}$ Aside from the specific properties following from quantum metamaterial being an extended quantum system, it is also a medium with an optical response, which can be changed at will without changing its microstructure; such media are a focus of strong research effort. ${ }^{25,26}$

In this paper we show that the local manipulation of the quantum states of the constituent units of a quantum metamaterial is not the only possible way of initializing it in a desirable state. To be specific, we show that a spatially periodic quantum state of a one-dimensional (1D) quantum metamaterial (i.e., quantum photonic crystal ${ }^{11}$ ) can be realized by sending, from opposite directions, a pair of priming pulses through the system. This provides an easier way to initialize a quantum metamaterial, since it would not require local control of each qubit in it. Disposing of this requirement significantly simplifies the experimental realization of quantum metamaterials by reducing the following: the complexity of their design, the coupling to the environment, and therefore the decoherence due to both internal and external sources.

We model a 1D quantum metamaterial using superconducting charge qubits, ${ }^{11}$ though the main idea of our approach is independent of the particular implementation. The (identical) charge qubits are formed by superconducting islands sandwiched between superconducting strips and separated from them by tunneling barriers (see Fig. 1). The metamaterial occupies the central ("active") section of the transmission line formed by the strips; in the rest of the system ("passive") qubits are replaced by capacitive elements chosen to optimize the impedance matching between the active and passive sections of the line.

\section{MODEL OF A QUANTUM METAMATERIAL}

As in Ref. 11, we treat the electromagnetic field in the system classically while quantizing the qubit degrees of freedom. The field propagates along the $z$ direction. Neglecting edge effects, the magnetic field will have only a $y$ component, and the vector potential in a section between the $n$th and $(n+1)$ st qubits can be chosen as $A_{x n} \hat{e}_{x}$. The energy of the active part of the system can be then written as

$$
\begin{aligned}
E_{S C}= & \sum_{n=1}^{n=N}\left\{\frac{E_{J}}{2 \omega_{J}^{2}}\left[\left(\frac{2 \pi D \dot{A}_{x n}}{\Phi_{0}}+\dot{\varphi}_{n}\right)^{2}+\left(\frac{2 \pi D \dot{A}_{x n}}{\Phi_{0}}-\dot{\varphi}_{n}\right)^{2}\right]\right. \\
& -E_{J}\left[\cos \left(\varphi_{n}+\frac{2 \pi D A_{x n}}{\Phi_{0}}\right)+\cos \left(\varphi_{n}-\frac{2 \pi D A_{x n}}{\Phi_{0}}\right)\right] \\
& \left.+\frac{D L_{0} W \dot{A}_{x n}^{2}}{8 \pi c^{2}}+\frac{D L_{0} W}{8 \pi}\left(\frac{A_{x, n+1}-A_{x, n}}{L_{0}}\right)^{2}\right\}
\end{aligned}
$$




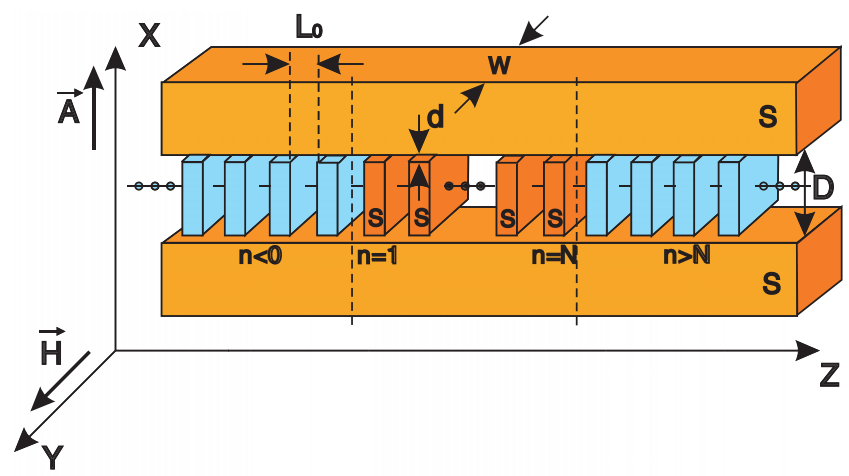

FIG. 1. (Color online) 1D quantum metamaterial in a superconducting transmission line. The superconducting islands $(S)$ sandwiched between the superconducting strips form charge qubits $(1 \leqslant n \leqslant N) ; D$ is the distance between the strips $(S)$, and $L_{0}$ is the distance between the qubits. In the passive (in and out) regions, with $n \leqslant 0$ and $n>N$, respectively, the transmission line parameters are chosen to optimize impedance matching with the active (metamaterial) section. The electromagnetic pulse propagates along the $z$ axis, $\vec{A}$ is the vector potential, and $\vec{H}$ is the magnetic field of the electromagnetic wave.

Here $\varphi_{n}$ is the superconducting phase of the $n$th island, $E_{J}=$ $\Phi_{0} I_{c} / 2 \pi c$ and $\omega_{J}^{2}=2 e I_{c} / \hbar C$ are the Josephson energy and Josephson frequency, respectively, $I_{c}$ is the critical current, $C$ is the Josephson junction capacity, $\Phi_{0}=h c / 2 e$ is the magnetic flux quantum, $D$ is the distance between the superconducting lines, and $W$ is the thickness of the superconducting strips. The first term in Eq. (1) is the charging energy of the $n$th qubit, the second is the Josephson energy, and the last two terms describe the electromagnetic field energy. The time derivative is indicated by a $\operatorname{dot}(\dot{A}, \dot{\varphi})$. We assume here that the qubit size is much smaller than the distance between the neighboring qubits and that the vector potential does not depend on the coordinates in the area between them. Similarly, in the passive regions

$$
\begin{aligned}
E_{N C}= & \sum_{n \leqslant 0, n>N}\left[\frac{\tilde{C} D^{2} \dot{A}_{x n}^{2}}{c^{2}}+\frac{D L_{0} W \dot{A}_{x n}^{2}}{8 \pi c^{2}}\right. \\
& \left.+\frac{D L_{0} W}{8 \pi}\left(\frac{A_{x, n+1}-A_{x, n}}{L_{0}}\right)^{2}\right] .
\end{aligned}
$$

Comparing the expressions for the electromagnetic field energy for the active [Eq. (1)] and the passive [Eq. (2)] regions one can conclude that the impedance matching is fulfilled if $\tilde{C}=4 e^{2} E_{J} / \hbar^{2} \omega_{J}^{2}$.

In order to obtain the Hamiltonian from the full classical energy of the system, $E_{S C}+E_{N C}$, we quantize the qubit contributions by writing

$$
\begin{aligned}
\hat{H}_{n}|n\rangle & =E_{n}|n\rangle, \\
\hat{H}_{n} & =-\frac{\left(\hbar \omega_{J}\right)^{2}}{E_{J}}\left(\frac{\partial}{\partial \varphi_{n}}\right)^{2}-2 E_{J} \cos \varphi_{n}+\hat{h}_{n} .
\end{aligned}
$$

Here the term $\hat{h}_{n}$ describes the external controls of the state of the $n$th qubit; its precise form is irrelevant here. Then we restrict the operator $\hat{H}_{n}$ to the subspace of its two lowest states, $\left|0_{n}\right\rangle$ and $\left|1_{n}\right\rangle$, while $E_{1, n}-E_{0, n}=\hbar \varepsilon_{n}$. We can assume this due to the nonlinearity of the Josephson potential, assuming that both the temperature and the field amplitudes are small enough to keep only these states populated.

As follows from Eq. (1), the interaction of the qubit with the electromagnetic field is given by

$$
\hat{V}_{n}=2 E_{J}\left(1-\cos a_{n}\right) \cos \varphi_{n},
$$

where $a_{n}=2 \pi D A_{x n} / \Phi_{0}$ is the dimensionless vector potential.

\section{EQUATIONS OF MOTION OF A QUANTUM METAMATERIAL}

In the following, we consider the simplest case of the factorizable wave function of the metamaterial. Each qubit is described by its own wave function $\left|\psi_{n}\right\rangle$ :

$$
\left|\psi_{n}(t)\right\rangle=c_{0}(n, t)|0\rangle e^{i \varepsilon t / 2}+c_{1}(n, t)|1\rangle e^{-i \varepsilon t / 2} ;
$$

we omit here the subscripts in $|0\rangle$ and $|1\rangle$, since the qubits are assumed to be identical. The Shrödinger equation for $\left|\psi_{n}\right\rangle$ is thus reduced to a set of equations for the coefficients $c_{0}$ and $c_{1}$ :

$$
i \hbar \dot{c}_{\alpha}(n, t)=\sum_{\beta=0,1}\left\langle\alpha\left|V_{n}(t)\right| \beta\right\rangle c_{\beta}(n, t) e^{i\left(\omega_{\alpha}-\omega_{\beta}\right) t},
$$

where $(\alpha, \beta)=0,1, \omega_{1}=\varepsilon / 2$, and $\omega_{0}=-\varepsilon / 2$. From Eq. (1) the equation for the electromagnetic field in the active region $1 \leqslant n \leqslant N$ follows:

$\ddot{a}_{n}-v^{2}\left(a_{n+1}+a_{n-1}-2 a_{n}\right)+r \sin a_{n}\left\langle\psi_{n}\left|\cos \varphi_{n}\right| \psi_{n}\right\rangle=0$,

where

$v^{2}=\frac{r W \Phi_{0}^{2}}{32 \pi^{3} L_{0} D E_{J}}, \quad r=\omega_{J}^{2}\left(1+\frac{\Phi_{0}^{2} L_{0} W \omega_{J}^{2}}{32 \pi^{3} c^{2} D E_{J}}\right)^{-1}$.

In the passive regions we have instead

$$
\ddot{a}_{n}-u^{2}\left(a_{n+1}+a_{n-1}-2 a_{n}\right)=0 .
$$

With the proper choice of parameters in the passive region one can obtain $u=v$.

For an analytical treatment, we make additional plausible assumptions. First, the electromagnetic field must be weak, $a_{n} \ll 1$ (otherwise the two-level approximation for qubits may not be valid); therefore

$$
\hat{V}_{n} \approx E_{J} a_{n}^{2} \cos \varphi_{n}
$$

and $\sin a_{n} \approx a_{n}$. Second, the field wavelength under a realistic choice of parameters greatly exceeds the interqubit distance and therefore allows to introduce a continuous variable $z$ instead of the discrete label $n$ (see Ref. 11). Then Eq. (7) is transformed into

$$
\frac{\partial^{2} a}{\partial t^{2}}-\tilde{v}^{2} \frac{\partial^{2} a}{\partial z^{2}}+\chi(z, t) a=0
$$

where $\tilde{v}=v L_{0}, a \equiv a(z, t)$, and the "susceptibility"

$$
\chi(z, t)=r\langle\psi(z, t)|\cos \varphi| \psi(z, t)\rangle
$$

is determined by the quantum state of the metamaterial. 


\section{INITIALIZATION OF A QUANTUM METAMATERIAL BY PRIMING PULSES}

In agreement with our weak-field assumption, we analyze Eq. (11) in a quasilinear approximation. These analytical results will help illuminate the numerical solutions of Eqs. (6), (7), and (9) for the following choice of parameters: $L_{0}=5 \times 10^{-4} \mathrm{~m}, D \sim W \sim 10^{-5} \mathrm{~m}$, critical current $I_{c}=4 \times 10^{-7} \mathrm{~A}, \varepsilon / 2 \pi \simeq 5 \times 10^{10} \mathrm{~s}^{-1}$, and $E_{J} / \hbar \omega_{J}=4$, so $\tilde{v} \simeq c$.

As shown in Ref. 11, the transmission properties of a 1D metamaterial with a spatially periodic quantum state, $\left|\psi\left(z+L_{m}, t=0\right)\right\rangle=|\psi(z, t=0)\rangle$, are those of a generalized photonic crystal. We therefore investigate the possibility of initializing the metamaterial without applying local controls to the constituent qubits. Specifically, let us initialize the metamaterial in the ground state (which can be done by, e.g., cooling) and send two priming pulses through the active region of the system in opposite directions. One would expect that due to the interference of these pulses and their action on the qubits, a spatially periodic state of the quantum metamaterial would arise. For superconducting qubits the decoherence times are currently in the range 10-100 $\mu$ s and the interlevel distance is $\sim 10 \mathrm{GHz}$; the transition time for the pulse across a 1000-unit metamaterial (of length $\sim 10^{2} \mathrm{~cm}$ ) is $\sim 3 \times 10^{-9} \mathrm{~s}$. Therefore, in the following, we can neglect decoherence effects.

The backaction of the electromagnetic field on the qubits is quadratic in the field amplitude [Eq. (10)], therefore the basic frequency of the priming pulses should satisfy the condition $2 \omega \approx \varepsilon$ (see, e.g., Refs. 11, 27, and 28). We assume that the spatial width of the pulse satisfies

$$
l \gg 2 \pi \tilde{v} / \omega \approx \lambda,
$$

which ensures that each qubit in a superposition state undergoes many cycles of quantum beats while the pulse propagates past it. Here $\lambda$ is the wavelength of the priming pulses. In this case, the metamaterial state evolution in the presence of the field can be investigated in the resonance approximation. ${ }^{27}$ So, Eqs. (6) become

$$
\begin{aligned}
& i \hbar \dot{c}_{0}(z, t)=a^{2}(z, t)\left(d_{00} c_{0}+d_{01} c_{1} e^{-i \varepsilon t}\right), \\
& i \hbar \dot{c}_{1}(z, t)=a^{2}(z, t)\left(d_{10} c_{0} e^{i \varepsilon t}+d_{11} c_{1}\right),
\end{aligned}
$$

where $d_{\alpha \beta}=E_{J}\left\langle\alpha\left|\cos \varphi_{n}\right| \beta\left\langle\right.\right.$ and $z=n L_{0}$. In the case of sufficiently strong pulses the solution of Eq. (11) may be written as

$$
\begin{aligned}
& a^{(1)}(z, t)=\exp \left[-\frac{[z-(\omega / k) t]^{2}}{l^{2}}\right]\left(A e^{i(k z-\omega t)}+\text { c.c. }\right), \\
& a^{(2)}(z, t)=\exp \left[-\frac{[z+(\omega / k) t]^{2}}{l^{2}}\right]\left(A e^{i\left(k z+\omega t+\phi_{0}\right)}+\text { c.c. }\right)
\end{aligned}
$$

where $A$ is the amplitude of the pulses (the pulses have equal amplitudes), and $\phi_{0}$ is the initial phase. In order for our treatment of the metamaterial as a continuous medium to be consistent, the wave vectors should satisfy the condition $\lambda=2 \pi / k \gg L_{0}$, that is,

$$
L_{0} \ll 2 \pi \tilde{v} / \omega .
$$

For $\tilde{v} \approx c$ and $\varepsilon / 2 \pi \approx 5 \times 10^{10} \mathrm{~s}^{-1}$, this yields $L_{0} \ll 1 \mathrm{~cm}$, which is a feasible requirement.

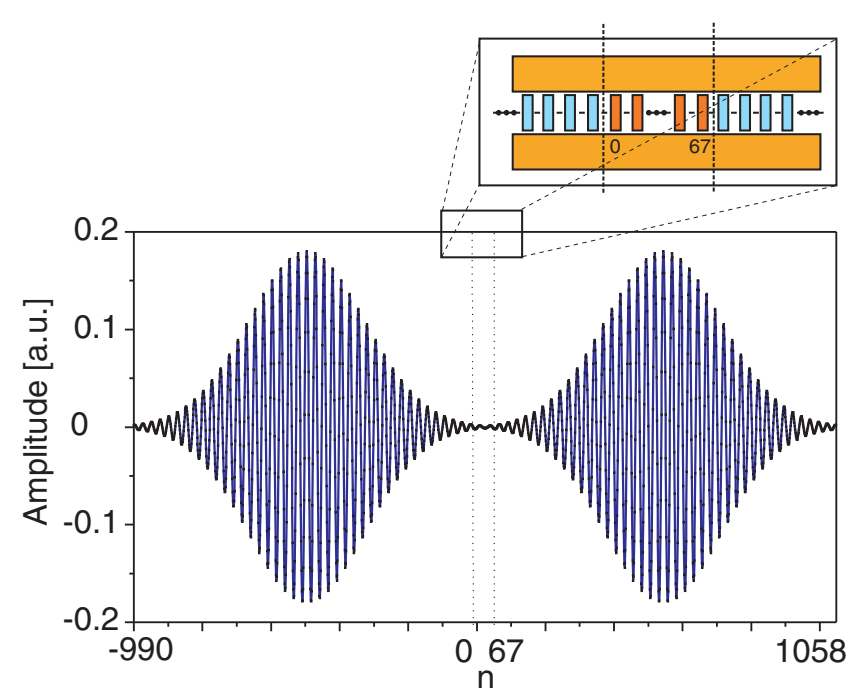

FIG. 2. (Color online) The priming or initializing pulses located in the passive regions of the waveguide line at the initial moment of time. The active region is in the center of the line between the two vertical dotted lines. The pulse parameters are $\tilde{v}=c$, $\omega=\varepsilon / 2, \Delta=0.18 \varepsilon, k=(1 / 25)\left(2 \pi / L_{0}\right)$. The pulse width is $l=$ $240 L_{0}$, and the pulse amplitude $A=0.18$. The matrix elements are $d_{00}=0.4 \hbar \varepsilon, d_{11}=3.6 \hbar \varepsilon$, and $d_{01}=0.2 \hbar \varepsilon$. The total length of the system simulated here is $\tilde{L}=2048 L_{0}$.

Let all the qubits be initialized at $t=0$ in the ground state $\left[c_{0}(z, 0)=1, c_{1}(z, 0)=0\right]$, then, at later times, the quasimonochromatic approximation [Eq. (13)] gives

$$
\begin{array}{r}
\left|c_{1}(z, t)\right|=\frac{|\Omega(z)| \sin \left(\sqrt{|\Omega(z)|^{2}+\gamma(z)^{2} / 4} t\right)}{\sqrt{|\Omega(z)|^{2}+\gamma(z)^{2} / 4}}, \\
\gamma(z)=\Delta+4 A^{2} \frac{d_{00}-d_{11}}{\hbar}\left[\cos \left(2 k z+\phi_{0}\right)+1\right],
\end{array}
$$

where $\Delta=2 \omega-\varepsilon$ is the detuning from the resonance. The local Rabi frequency is

$$
|\Omega(z)|=\frac{2\left|d_{01}\right| A^{2}}{\hbar}\left[\cos \left(2 k z+\phi_{0}\right)+1\right]
$$

The periodicity of $\gamma(z)$ and $\Omega(z)$ [Eqs. (18) and (19)] implies a spatially periodic probability of exciting the qubit with period $\lambda / 2$.

Figures 2 and 3 show the results of a numerical simulation of such a process from Eqs. (7) and (9). Two priming pulses at $t=0$ are located in the passive regions of the waveguide (Fig. 2). After passing through the active region, they produce a periodically modulated population of the levels of the qubits (Fig. 3). A small distortion of the periodicity is due to the fact that the waves are nonmonochromatic and can be improved by using wider pulses. For the system parameters chosen, the period of modulation in Fig. 3 is approximately equal to $13 L_{0}$, in good agreement with the modulation period $\pi / k=$ $12.5 L_{0}$ following from the approximate solution Eq. (19). The modulation amplitude can also be controlled by the pulse amplitude and the pulse width [cf. Eqs. (17) and (19)]. 


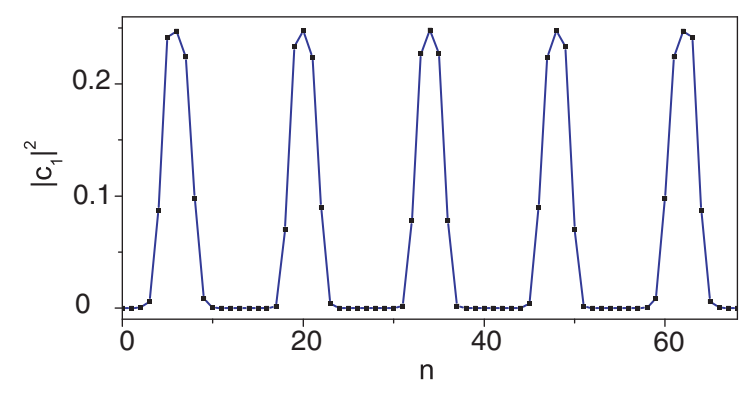

FIG. 3. (Color online) Periodically modulated population of the excited levels of qubits after passing the priming or initializing pulses through the system. The period of modulation, $13 L_{0} \approx \lambda / 2$, shows that this is due to the interference between these pulses. The slight aperiodicity is caused by the finite width of the pulse.

\section{PULSE PROPAGATION THROUGH A QUANTUM METAMATERIAL}

The periodic spatial modulation of the wave function $|\psi(z)\rangle$, created by the priming pulses, will affect the propagation of the subsequent probe pulse through the function $\chi(z)$ [Eq. (12)], which enters the wave equation (11):

$$
\begin{aligned}
\chi(z, t)= & \chi_{0}+\tilde{\chi}\left[1+\cos \left(2 \pi z / L_{m}\right)\right] \\
& +2 \frac{r}{E_{J}} \operatorname{Re}\left[d_{01} c_{0}^{*}(z) c_{1}(z) e^{-i \varepsilon t}\right],
\end{aligned}
$$

where $L_{m}$ is the modulation period, and $\chi_{0}$ depends on the quantum states of the qubits with minimal $\left|c_{1}\right|$ :

$$
\chi_{0}=\frac{r}{E_{J}}\left(d_{00}\left|c_{0, \min }\right|^{2}+d_{11}\left|c_{1, \min }\right|^{2}\right) .
$$

For the case shown in Fig. 3, $\chi_{0}=\left(r / E_{J}\right) d_{00}$, since the qubits at positions such that $\cos \left(2 k z+\phi_{0}\right)=-1$ (i.e., where the local Rabi frequency is zero) remain in the ground state, and thus $\left|c_{1, \min }\right|=0$. The quantity $\tilde{\chi}$ describes the excitation of qubits at positions where $\cos \left(2 k z+\phi_{0}\right)=1$, and $\left|c_{1}\right|=\left|c_{1, \max }\right|$ :

$$
\tilde{\chi}=r\left(d_{11}-d_{00}\right)\left(\left|c_{1, \max }\right|^{2}-\left|c_{1, \min }\right|^{2}\right) / 2 E_{J} .
$$

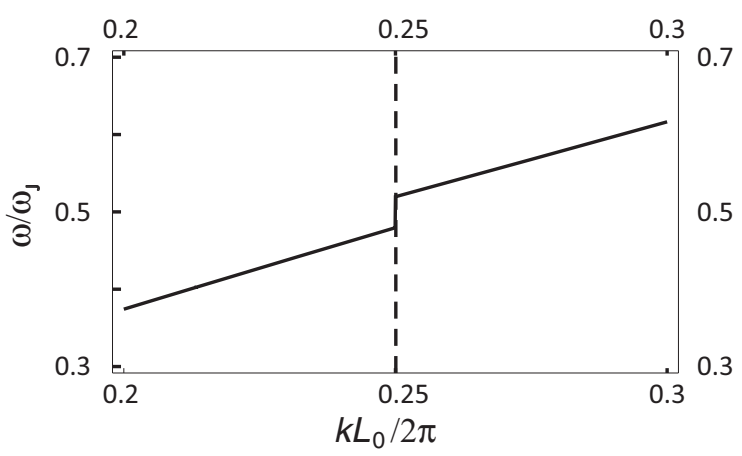

FIG. 4. Normalized dispersion relation $\omega(k)$ for the quantum photonic crystal. Due to the space periodicity of $\chi(z)$, gaps open in the spectrum. Their positions and magnitude depend on $L_{m}$ and $\tilde{\chi}$. This allows to control the parameters of the quantum photonic crystal by varying the quantum states of the qubits. The graph is plotted for $L_{m}=25 L_{0} / 2, d_{00}=0.4 \hbar \varepsilon, d_{11}=3.6 \hbar \varepsilon, d_{01}=0.2 \hbar \varepsilon$, and $\tilde{v}=c$. The qubit population is the same as in Fig. 3.
For the case of Fig. 3 we have $\tilde{\chi}=r\left(d_{11}-d_{00}\right)\left|c_{1, \max }\right|^{2} / 2 E_{J}$. If

$$
\tilde{\chi} \gg\left|\int_{-L_{m} / 2}^{L_{m} / 2}\left[\left(r / E_{J}\right) d_{01} c_{0}^{*}(z) c_{1}(z) e^{i 2 \pi z / L_{m}}\right] d z\right|,
$$

then one can neglect the third term in Eq. (20) to derive the dispersion equation and thus consider $\chi$ as independent of time. So, $\chi$ in Eq. (20) can be approximated as

$$
\chi(z)=\chi_{0}+\tilde{\chi}\left[1+\cos \left(2 \pi z / L_{m}\right)\right] .
$$

We seek the solution of Eq. (11) with the above $\chi(z)$ for a small wave amplitude in the form of a Bloch wave: ${ }^{29}$

$$
a_{k}(z, t)=u_{k}(z) \exp [i(k z-\omega t)]
$$

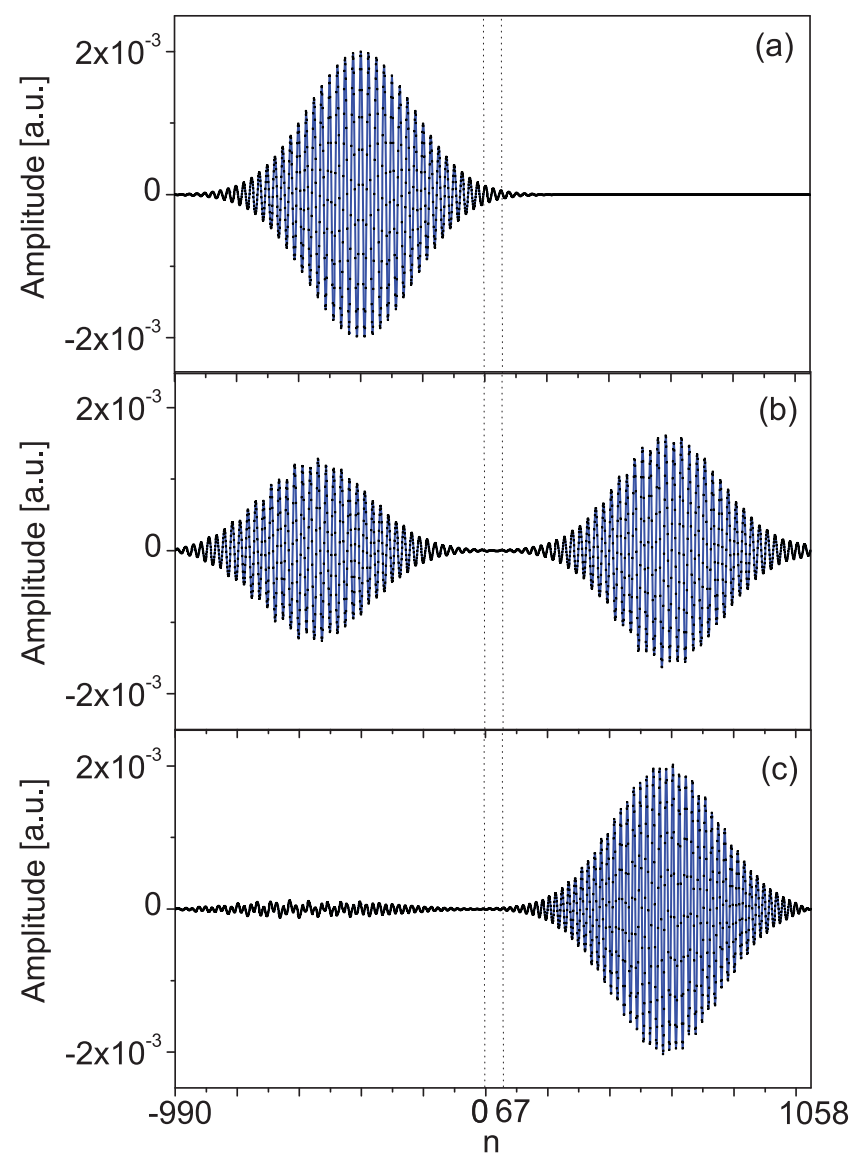

FIG. 5. (Color online) Probe pulse propagation through a spatially periodic quantum metamaterial prepared by the priming pulses. The vertical dotted lines indicate the location of the chain of qubits (i.e., the actual metamaterial). The metamaterial parameters are the same as in Fig. $2 ; d_{00}=0.4 \hbar \varepsilon, d_{11}=3.6 \hbar \varepsilon, d_{01}=0.2 \hbar \varepsilon$; the qubit population is the same as in Fig. 3. (a) The probe pulse at $t=0$. The pulse parameters are as follows: amplitude $A=2 \times 10^{-3}$, width $l=240 L_{0}$, carrier frequency $\omega=\varepsilon / 2$, and velocity $\tilde{v}=c$. (b) Field distribution at $t=500 \varepsilon^{-1}$. The partial pulse transmission is due to the finite pulse width. (c) Field distribution at $t=500 \varepsilon^{-1}$ for a probe pulse with frequency above the band gap. The pulse parameters at $t=0$ are the same as above, except the carrier frequency now is $\omega=0.6 \varepsilon$. 
where $u_{k}(z)$ is a periodic function with period $L_{m}$ [i.e., $u_{k}(z)=$ $\left.u_{k}\left(z+L_{m}\right)\right]$. The dispersion equation is readily found:

$$
\omega_{k}^{2}-\tilde{v}^{2} k^{2}= \pm\left|W_{k}\right|+W_{k=0}
$$

where

$$
W_{k}=\frac{1}{L_{m}} \int_{-L_{m} / 2}^{+L_{m} / 2} \chi(z) \exp (i 2 k z) d z, \quad k=\frac{\pi n}{L_{m}} .
$$

It is clear from Eq. (25) that a gap opens in the frequency spectrum for each $k=\pi n / L_{m}$ [if the corresponding Fourier component of $\chi(z)$ is different from zero; see Fig. 4]:

$$
\delta \omega_{n} \approx \frac{W_{k_{n}}}{\sqrt{\left(\pi \tilde{v} n / L_{m}\right)^{2}+W_{0}}}, \quad\left|W_{k_{n}}\right| \ll\left(\frac{\pi \tilde{v} n}{L_{m}}\right)^{2}+W_{0} .
$$

The gap appears for $k_{n=1}=\pi / L_{m}$, close to the frequency $\omega_{1}=\sqrt{\left(\tilde{v} k_{1}\right)^{2}+W_{0}}$. According to Eq. (26), $W_{k_{n=1}}=\tilde{\chi} / 2$, $W_{0}=\chi_{0}+\tilde{\chi}$, and the gap equals

$$
\delta \omega_{1} \approx \tilde{\chi} / \sqrt{\left(\pi \tilde{v} / L_{m}\right)^{2}+W_{0}} .
$$

In Fig. 5 the propagation of a probe pulse through a metamaterial with periodic $\chi(z)$ is shown. One can see that while the pulse with the carrying frequency inside the band gap undergoes significant reflection [Fig. 5(a)], this is not the case for a pulse with a frequency above the band gap [Fig. 5(b)].

\section{CONCLUSIONS}

In conclusion, we have shown that a $1 \mathrm{D}$ quantum metamaterial comprising superconducting charge qubits in a transmission line can be initialized in a spatially periodic state without exercising local control of the quantum state of individual qubits, by simultaneously passing through it two priming pulses in opposite directions. The modulation period is close to half the priming pulse wavelength, $\lambda / 2$. The subsequent probe pulse propagation through the resulting periodic structure was shown to be affected by the arising band gaps.

It is possible that such $1 \mathrm{D}$ quantum metamaterials could be realized using the microwave transmission line that was recently experimentally investigated in Ref. 30, where a set of resonators coupled with qubits was connected with this line. If the transmission line is long enough, we can create a more complex picture of the qubit state distribution by varying the shape of the pump pulses.

\section{ACKNOWLEDGMENTS}

This work was funded in part by the Russian Ministry of Education and Science through the programs (No. 07.514.11.4147 and No. 14.B37.21.0079), and the Russian Foundation for Basic Research (Grants No. 12-07-00546 and No. 12-07-31144). F.N. was partially supported by the ARO, RIKEN iTHES project, JSPS-RFBR Contract No. 1202-92100, Grant-in-Aid for Scientific Research (S), MEXT Kakenhi on Quantum Cybernetics, and the JSPS via its FIRST program. S.S. and A.Z. acknowledge that this publication was made possible through the support of a grant from the John Templeton Foundation. *alexshdze@mail.ru

†a.zagoskin@1boro.ac.uk

${ }^{1}$ V. G. Veselago, Sov. Phys. Usp. 46, 764 (2003).

${ }^{2}$ J. B. Pendry, A. J. Holden, D. J. Robbins, and W. J. Stewart, IEEE Trans. Microwave Theory Tech. 47, 2075 (1999).

${ }^{3}$ R. A. Shelby, D. R. Smith, and S. Schultz, Science 292, 77 (2001).

${ }^{4}$ W. J. Padilla, D. N. Basov, and D. R. Smith, Mater. Today 9, 28 (2006).

${ }^{5}$ K. Y. Bliokh, Y. P. Bliokh, V. Freilikher, S. Savel'ev, and F. Nori, Rev. Mod. Phys. 80, 1201 (2008).

${ }^{6}$ M. Ricci, N. Orloff, and S. M. Anlage, Appl. Phys. Lett. 87, 034102 (2005).

${ }^{7}$ C. G. Du, H. Y. Chen, and S. Q. Li, Phys. Rev. B 74, 113105 (2006).

${ }^{8}$ S. Savel'ev, A. L. Rakhmanov, and F. Nori, Phys. Rev. Lett. 94, 157004 (2005); S. Savel'ev, V. A. Yampol'skii, A. L. Rakhmanov, and F. Nori, Rep. Prog. Phys. 73, 026501 (2010).

${ }^{9}$ V. A. Yampol'skii, S. Savel'ev, O. V. Usatenko, S. S. Mel'nik, F. V. Kusmartsev, A. A. Krokhin, and F. Nori, Phys. Rev. B 75, 014527 (2007).

${ }^{10}$ N. Lazarides and G. P. Tsironis, Appl. Phys. Lett. 90, 163501 (2007).

${ }^{11}$ A. L. Rakhmanov, A. M. Zagoskin, S. Savel'ev, and F. Nori, Phys. Rev. B 77, 144507 (2008).

${ }^{12}$ D. Burgarth, K. Maruyama, and F. Nori, New J. Phys. 13, 013019 (2011).
${ }^{13}$ D. Burgarth, K. Maruyama, and F. Nori, Phys. Rev. A 79, 020305(R) (2009).

${ }^{14}$ D. Burgarth, K. Maruyama, M. Murphy, S. Montangero, T. Calarco, F. Nori, and M. B. Plenio, Phys. Rev. A 81, 040303(R) (2010).

${ }^{15}$ A. M. Zagoskin, A. L. Rakhmanov, S. Savel'ev, and F. Nori, Phys. Status Solidi B 246, 955 (2009).

${ }^{16}$ N. I. Zheludev, Science 328, 582 (2010).

${ }^{17}$ D. Felbacq, J. Nanophoton. 5, 050302 (2011).

${ }^{18}$ C. Hutter, E. A. Tholen, K. Stannigel, J. Lidmar, and D. B. Haviland, Phys. Rev. B 83, 014511 (2011).

${ }^{19}$ A. M. Zagoskin, Quantum Engineering: Theory and Design of Quantum Coherent Structures (Cambridge University Press, Cambridge, UK, 2011).

${ }^{20}$ J. Q. You and F. Nori, Nature (London) 474, 589 (2011); Phys. Today 58(11), 42 (2005).

${ }^{21}$ J. Q. Quach, Ch.-H. Su, A. M. Martin, A. D. Greentree, and Lloyd C. L. Hollenberg, Opt. Express 19, 11018 (2011).

${ }^{22}$ D. Zueco, J. J. Mazo, E. Solano, and J. J. Garcia-Ripoll, Phys. Rev. B 86, 024503 (2012).

${ }^{23}$ A. M. Zagoskin, J. Opt. 14, 114011 (2012).

${ }^{24}$ O. Astafiev, A. M. Zagoskin, A. A. Abdumalikov, Jr., Yu. A. Pashkin, T. Yamamoto, K. Inomata, Y. Nakamura, and J. S. Tsai, Science 327, 840 (2010).

${ }^{25}$ L. Zhou, Z. R. Gong, Y. X. Liu, C. P. Sun, and F. Nori, Phys. Rev. Lett. 101, 100501 (2008); L. Zhou, H. Dong, Y. X. Liu, C. P. Sun, and F. Nori, Phys. Rev. A 78, 063827 (2008). 
${ }^{26}$ A. Gelman and Y. V. Radeonychev, J. Phys. B 43, 155402 (2010).

${ }^{27}$ V. M. Fain and Ya. I. Khanin, Quantum Electronics (MIT Press, Cambridge, MA, 2003).

${ }^{28}$ L. Allen and J. H. Eberly, Optical Resonance and Two-Level Atoms (Wiley, New York, 1975).
${ }^{29}$ N. W. Ashcroft and N. D. Mermin, Solid State Physics (Harcourt, Orlando, FL, 1976).

${ }^{30}$ M. Jerger, S. Poletto, P. Macha, U. Huebner, A. Lukashenko,

E. Il'ichev, and A. V. Ustinov, Europhys. Lett. 96, 40012 (2011). 